\title{
Nuclear quantum effect for hydrogen adsorption on $\operatorname{Pt}(111)$
}

\author{
Lei Yan $\odot,{ }^{1,2, *}$ Yoshiyuki Yamamoto, ${ }^{2}$ Motoyuki Shiga, ${ }^{3}$ and Osamu Sugino ${ }^{2}$ \\ ${ }^{1}$ School of Physics and Information Technology, Shaanxi Normal University, Xi'an 710119, China \\ ${ }^{2}$ Institute for Solid State Physics, The University of Tokyo, 5-1-5 Kashiwanoha, Kashiwa, Chiba 277-8581, Japan \\ ${ }^{3}$ CCSE, Japan Atomic Energy Agency (JAEA), 178-4-4, Wakashiba, Kashiwa, Chiba 277-0871, Japan
}

(Received 9 January 2020; accepted 16 March 2020; published 16 April 2020)

\begin{abstract}
Nuclear quantum effect and many-body interaction importantly interplay in the hydrogen on the $\operatorname{Pt}(111)$ system under the high coverage conditions of electrochemical interest, as revealed by our ab initio path integral and ring polymer molecular dynamics simulations done at room temperature. At the full monolayer coverage, hydrogen atoms are close-packed either at the atop sites or the fcc sites owing to their strong repulsion and the nearly degenerate nature of the adsorption sites. While at the $2 / 3$ monolayer, they are delocalized over the fcc and hcp sites via the bridge sites because of the hopping. The quantum many-body effect is thus crucially important in determining the coverage dependence and provides a clue for reconciling the long-standing controversy on this system.
\end{abstract}

DOI: 10.1103/PhysRevB.101.165414

\section{INTRODUCTION}

Hydrogen $(\mathrm{H})$ adsorption on metal electrodes is one of the most fundamental issues in electrochemistry for decades [1]. This problem has been studied extensively as a first step for understanding the hydrogen evolution and oxidation reactions (HER and HOR) in hydrogen-based fuel cells, where platinum $(\mathrm{Pt})$ is commonly used as the electrocatalyst. Experiments have been carried out to solve this issue by preparing single crystal surfaces interfaced with solution, but it is not clear yet which is the most stable adsorption sites even for the most-stable and close-packed (111) surface of Pt [2-7]. The energetically favorable adsorption sites for $\mathrm{Pt}(111)$ usually include $\mathrm{H}$ adsorbed on the fcc, hcp, and atop sites $\left(\mathrm{H}_{\mathrm{fcc}}\right.$, $\mathrm{H}_{\text {hcp }}$, and $\mathrm{H}_{\text {atop }}$ ), where both $\mathrm{H}_{\text {fcc }}$ and $\mathrm{H}_{\text {hcp }}$ belong to the hollow site. Depending on the electrode potential, adsorbed $\mathrm{H}\left(\mathrm{H}^{*}\right)$ is classified into two types: underpotentially deposited $\mathrm{H}\left(\mathrm{H}_{\mathrm{upd}}\right)$ at positive potential relative to the onset of HER and overpotentially deposited $\mathrm{H}\left(\mathrm{H}_{\mathrm{opd}}\right)$ at the negative potential. For $\mathrm{Pt}(111), \mathrm{H}_{\text {upd }}$ is usually believed to be adsorbed on the fcc site for the coverage up to one monolayer, while $\mathrm{H}_{\text {opd }}$ appears subsequently at atop site [5]. However, spectroscopy experiments reported the signal of $\mathrm{H}_{\text {atop }}$ emerging at the positive potential and the results are still inconclusive [6]. Evidently, a lack of knowledge about the structures of $\mathrm{H}_{\text {upd }}$ and $\mathrm{H}_{\text {opd }}$ limits our understanding about the properties of the electric double layer at equilibrium conditions and during HER [4].

The complicated aspect of the $\mathrm{H}$ adsorption at the atomic level has been elaborated using $a b$ initio simulations based on density functional theory (DFT) $[8,9]$. Simulations at the level of generalized gradient approximation (GGA) showed that $\mathrm{H}_{\mathrm{fcc}}$ is favored over $\mathrm{H}_{\text {atop }}$ at the full coverage by about $50 \mathrm{meV}[10,11]$, which is beyond the accuracy of DFT-GGA as can be deduced from the $\mathrm{CO}$ adsorption on $\mathrm{Pt}(111)$ [12]. More recently, we have revisited this problem with a higher

*Corresponding author: yanlei@snnu.edu.cn level of theory based on random phase approximation (RPA) [13]. With a critical convergence study, RPA calculations showed that $\mathrm{H}_{\mathrm{fcc}}$ is favored over the $\mathrm{H}_{\text {atop }}$ at the full coverage by about $23 \mathrm{meV}$ when the experimental lattice constant is used, predicting the coexistence of $\mathrm{H}_{\mathrm{fcc}}$ and $\mathrm{H}_{\text {atop }}$ at room temperature. In addition, the small energy differences in the previous work are mainly due to the difference in the zeropoint vibrational energy (ZPE) correction within the harmonic approximation. However, potential energy surface for $\mathrm{H}$ on $\mathrm{Pt}(111)$ is very flat [14], and it is desirable to explicitly include the anharmonic contribution to the nuclear quantum effect to resolve the $\mathrm{H}$ adsorption problem.

One approach taking account of nuclear quantum effect is path integral molecular dynamics (PIMD), which is based on Feynman's imaginary-time path integral (PI) formulation of quantum statistical mechanics $[15,16]$. Quantum statistics of a particle is treated as classical statistics of a ring polymer coupled by harmonic springs in PI theory within the secondorder expansion [17]. The quantum partition function of $N$ atoms is described as

$$
\begin{aligned}
Z= & \lim _{P \rightarrow \infty} \prod_{I=1}^{N}\left[\left(\frac{M_{I} P}{2 \pi \beta \hbar^{2}}\right)^{\frac{3 P}{2}} \int d \mathbf{R}_{I}^{(1)} \int d \mathbf{R}_{I}^{(2)} \cdots \int d \mathbf{R}_{I}^{(P)}\right] \\
& \times \exp \left[-\beta V_{\mathrm{eff}}\right]
\end{aligned}
$$

with the effective potential

$$
\begin{aligned}
V_{\mathrm{eff}}= & \sum_{s=1}^{P}\left\{\sum_{I=1}^{N} \frac{M_{I}}{2} \omega_{P}^{2}\left(\mathbf{R}_{I}^{(s)}-\mathbf{R}_{I}^{(s-1)}\right)^{2}\right. \\
& \left.+\frac{1}{P} V\left(\mathbf{R}_{1}^{(s)}, \ldots, \mathbf{R}_{N}^{(s)}\right)\right\},
\end{aligned}
$$

where $\mathbf{R}_{I}$ and $M_{I}$ are the position and mass of the $I$-th atom, respectively. Accordingly, the quantum statistics for a system including many atoms can be obtained from the classical 
ensemble generated by the molecular dynamics (MD) simulation of a system with many ring polymers composed of $P$ beads interconnected by harmonic springs with a frequency $\omega_{P}=\frac{\sqrt{P}}{\beta \hbar}[18,19]$. The ring polymer molecular dynamics (RPMD) [20] is a method that extends the concept of PIMD to approximate semiclassical dynamics via the linear response theory based on Kubo-transformed time correlation functions. Combined with electronic structure calculations of the BornOppenheimer potential energy $V$ and the relevant nuclear forces, these methods are generalized as ab initio PIMD and RPMD [21-25], where nuclear quantum effects related to the proton transfer [26], isotope effect [27], hydrogen diffusion in bulk metals $[28,29]$, dense hydrogen [30], hydrogen bonded crystals [31], water at metal surface [32], have been studied. However, on-the-fly $a b$ initio PIMD and RPMD techniques have never been used to elucidate hydrogen absorption sites on metal surfaces.

In this study, we apply the $a b$ initio PIMD and RPMD to $\mathrm{H}$ adsorption on $\operatorname{Pt}(111)$ at the coverage of 1 and 2/3. By comparing the results of $a b$ initio MD calculations, we show how the nuclear quantum effect affects the adsorption feature near the full coverage. By making a distinction between harmonic and anharmonic vibrational contributions due to the nuclear quantum effect, we show that the anharmonic one is also important at room temperature. In addition, the quantum effect is also analyzed by deriving an effective Hamiltonian for hydrogen atoms on the lattice, which reveals how the strongly repulsive interaction causes the coverage dependence. We conclude that the quantum many-body effect, which has been missing in previous theoretical studies, is the key to resolve the long-standing controversy about the coverage dependence observed in experiment.

\section{METHOD OF CALCULATION}

All the calculations were performed using the PIMD code [33], combined with the VASP code [34]. The electronic structure calculations were based on the projector-augmented wave [35] method and the GGA functional developed by Perdew, Burke, and Ernzerhof (PBE) [36]. In our previous work [13], the relative adsorption energy of $\mathrm{H}_{\mathrm{fcc}}$ and $\mathrm{H}_{\text {atop }}$ calculated with RPA was reproduced better with PBE than with van der Waals density functional. PBE is therefore used in this work because of reliability of RPA. Pt(111) substrate was modeled by a three-layer slab where all the layers was given full degrees of freedom to move. The plane wave basis functions are employed with a cutoff at $400 \mathrm{eV}$, and Brillouin zone is sampled by a $k$-points grid of $5 \times 5 \times 1$ with unit cell $\sqrt{3} \times \sqrt{3}$. We study the $\operatorname{Pt}(111)$ in vacuum and $\mathrm{Pt}(111)$ interfaced with two water bilayers (Fig. 1), the latter including two water molecules per layer and per unit cell. Two honeycomb lattices of water are stacked directly against each other, but with the water molecules staggered in terms of their relative orientations, as was done previously by ab initio MD simulations [37]. Note that water layers on $\mathrm{Pt}(111)$ in an UHV chamber typically desorb at temperatures below $200 \mathrm{~K}$ [38]. In order to prohibit the water layers from desorbing in our computational setup, the position of oxygen atoms of the outermost bilayer was fixed. We define $\theta$ as the coverage of
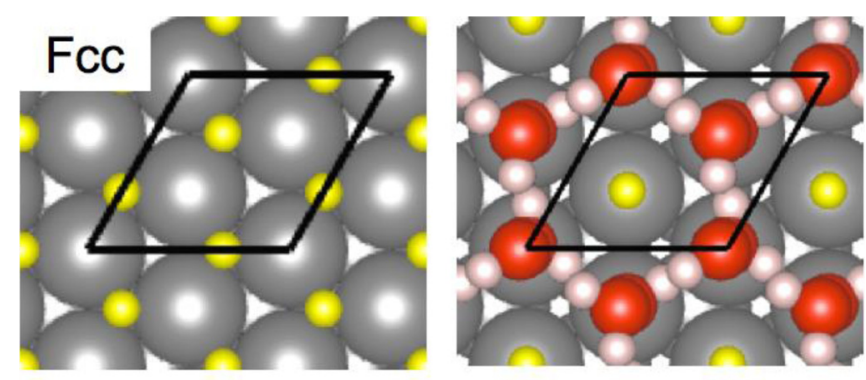

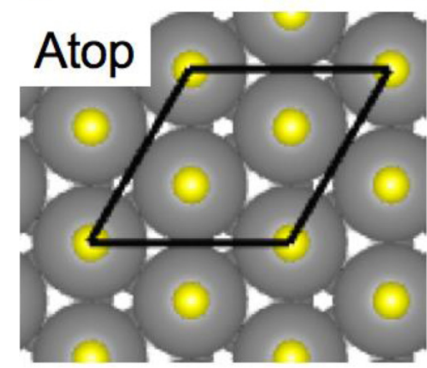

(a)

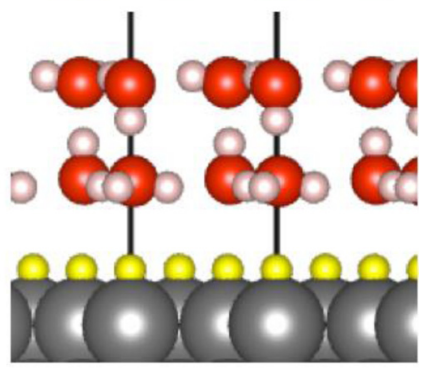

(b)
FIG. 1. Initial structure for $\mathrm{H}_{\mathrm{fcc}}$ and $\mathrm{H}_{\text {atop }}$ on $\mathrm{Pt}(111)$ at full coverage in vacuum (a), and covered with two water bilayers (b) in the top view (upper) and side view (lower) for $\mathrm{H}_{\text {atop }}$. The red, grey, and pink spheres represent oxygen, platinum, and hydrogen atoms, respectively. The hydrogen overlayer is drawn using yellow in order to understand the figures better.

$\mathrm{H}$ adsorption on $\mathrm{Pt}(111)$. In the following, the presence and absence of water bilayers are referred to as "solvated" and "vacuum", respectively.

The $a b$ initio MD and PIMD calculations were carried out at temperature of $300 \mathrm{~K}$ based on the Born-Oppenheimer scheme with the self-consistent-field convergence criteria of $1.0 \times 10^{-5} \mathrm{eV}$. To compute the energies and structures at thermal equilibria, we performed the $a b$ initio MD and PIMD calculations with the massive Nosé-Hoover chain (NHC) thermostats [39-41] attached to each degree of freedom to efficiently generate the canonical ensemble. To compute the vibrational spectra, $a b$ initio MD and RPMD calculations at constant energies were started from the equilibrated structures obtained from the thermostatted $a b$ initio MD and PIMD runs at $300 \mathrm{~K}$. The number of beads for ab initio PIMD and RPMD were set to be 16 , which is on the edge of convergence for path integral simulations of water at room temperature with respect to the energy and interatomic distributions [42-44]. The multiple time scale technique with the reversible reference system propagator algorithm was employed to integrate the NHC thermostats five times per time step [45]. The computational details of PIMD and RPMD simulations can be found in a recent review paper [19]. A $b$ initio MD and PIMD simulations were performed for $18-45 \mathrm{ps}$, which is insufficiently long to achieve the equilibrium but is enough to tentatively estimate the statistical error for adsorption energy less than $15 \mathrm{meV}$ (Table I). The time step was chosen as $0.25 \mathrm{fs}$, which is short enough to solve the equations of motion for the vibrations of adsorbed hydrogen on $\operatorname{Pt}(111)$ surface and liquid water [27,31]. The statistical convergence of the results has been checked by the block averages from two 
TABLE I. Calculated adsorption energy, temperature effect, and nuclear quantum effect from the static, classical, and quantum simulations. Unit in meV.

\begin{tabular}{lcccccrrr}
\hline \hline & & $E_{\text {ads }}^{\text {pimd }}$ & $E_{\text {ads }}^{\mathrm{md}}$ & $E_{\text {ads }}^{\text {opt }}$ & $\Delta E_{\text {ads }}^{\mathrm{q}}$ & $\Delta E_{\text {ads }}^{\mathrm{T}}$ & $\Delta E_{\text {ads }}^{\mathrm{H}}$ & $\Delta E_{\text {ads }}^{\mathrm{AH}}$ \\
\hline Vacuum & $\mathrm{H}_{\mathrm{fcc}}$ & $376 \pm 9$ & $452 \pm 7$ & 540 & $-76 \pm 16$ & $-88 \pm 7$ & -66 \\
& $\mathrm{H}_{\text {atop }}$ & $378 \pm 10$ & $495 \pm 6$ & 577 & $-117 \pm 16$ & $-82 \pm 6$ & -116 \\
Solvated & $\mathrm{H}_{\mathrm{fcc}}$ & $373 \pm 11$ & $458 \pm 7$ & 547 & $-85 \pm 18$ & $-89 \pm 7$ & $-69 \pm 10$ \\
& $\mathrm{H}_{\text {atop }}$ & $364 \pm 13$ & $489 \pm 7$ & 585 & $-126 \pm 20$ & $-95 \pm 7$ & -117 & $-105 \pm 11$ \\
& &
\end{tabular}

halves of the production runs. For convenience, $a b$ initio MD and $a b$ initio PIMD (RPMD) simulations are hereafter called classical and quantum simulations, respectively.

\section{RESULTS AND DISCUSSION}

Here, those surfaces adsorbed by $\mathrm{H}_{\mathrm{fcc}}$ and $\mathrm{H}_{\text {atop }}$ were chosen as the initial configurations of the dynamics runs, and they are named as "initial- $\mathrm{H}_{\mathrm{fcc}}$ " and "initial- $\mathrm{H}_{\mathrm{atop}}$ ", respectively. We note that the fcc site has been found to be the most stable adsorption site in the previous studies utilizing RPA and GGA $[10,11,13]$, while experiments suggest that the atop $\mathrm{H}$ plays a crucial role in the HOR/HER and is detected as mentioned above. We will show below the results for $\theta=1$ and $2 / 3$ on the vacuum and solvated surfaces, and analyze the importance of nuclear quantum effect for the hydrogen adsorption problem.

Adsorption at full coverage. Using the surface exposed to the vacuum [Fig. 1(a)], the pair distribution of the $\mathrm{Pt}-\mathrm{H}$ distances is obtained as shown in Fig. 2. In the classical simulation of the initial- $\mathrm{H}_{\mathrm{fcc}}$, the distribution shows two peaks at 1.56 and $1.78 \AA$, which correspond to the $\mathrm{H}_{\text {atop }}$ and $\mathrm{H}_{\mathrm{fcc}}$, respectively. These peaks can be compared with the $\mathrm{Pt}-\mathrm{H}$ distances of 1.56 and $1.86 \AA$ obtained by optimizing the geometry for the atop and fcc sites, respectively. That is, the $\mathrm{Pt}-\mathrm{H}_{\mathrm{fcc}}$ distance was shortened by the temperature effect while the $\mathrm{Pt}-\mathrm{H}_{\text {atop }}$ was not. During the first $15 \mathrm{ps}, \mathrm{H}^{*}$ hopped between the fcc and hcp sites more than ten times, as can be expected from the small barrier of $\sim 40 \mathrm{meV}$ previously shown to exist between these sites [46]. Afterwards, $\mathrm{H}^{*}$ transferred to atop site, as was reported in the foregoing classical simulation [47], and stayed there for the subsequent $30 \mathrm{ps.} \mathrm{When} \mathrm{starting}$ the classical simulation from the initial- $\mathrm{H}_{\text {atop }}$, the $\mathrm{Pt}-\mathrm{H}$ pair distribution only shows one peak at the position of $1.56 \AA$ in Fig. 2(b), indicating that $\mathrm{H}^{*}$ always stayed on the atop site. The occurrence of one event for the fcc-to-atop transfer is consistent with the larger (by $43 \mathrm{meV}$ ) adsorption energy of atop and the existing energy barrier, which was previously estimated to be $\sim 0.2 \mathrm{eV}$ [46]. Using the classical trajectory started from the initial- $\mathrm{H}_{\text {atop }}$, the density profile of $\mathrm{H}^{*}$ is obtained as shown in Fig. 2(c).

In the quantum simulation, the $\mathrm{Pt}-\mathrm{H}$ pair distributions display two peaks at 1.59 and $1.82 \AA$ similarly for both initial$\mathrm{H}_{\mathrm{fcc}}$ and initial- $\mathrm{H}_{\mathrm{atop}}$, which are stretched compared with classical simulation by 0.03 and $0.04 \AA$, respectively. Note that the distribution in the quantum case is much broader than that in the classical case as expected from the nuclear quantum effect. In addition, the adsorption energy averaged over the trajectory of quantum simulation is almost the same for the fcc and atop sites (see Table I). The similar distributions obtained from the two different initial geometries and the similar value of the adsorption energy suggest the coexistence of $\mathrm{H}_{\mathrm{fcc}}$ and $\mathrm{H}_{\text {atop }}$ structures, which is consistent with the prediction using the RPA energy with the ZPE correction [13]. Based on this discussion, the density profile is obtained from the initial- $\mathrm{H}_{\mathrm{fcc}}$ as shown in Fig. 2(c).

To consider the effect of the electrochemical environment, we also carried out a simulation including the water bilayers. The resulting pair distributions are shown in Fig. S1 [48]. We find that $\mathrm{H}^{*}$ always stayed at the initial site and no transfer occurred between fcc and atop site within 20 ps both for the classical and quantum simulations. The adsorption energy is larger for fcc only by $9 \mathrm{meV}$ for the quantum case, while the difference is $31 \mathrm{meV}$ for the classical case (Table I). These

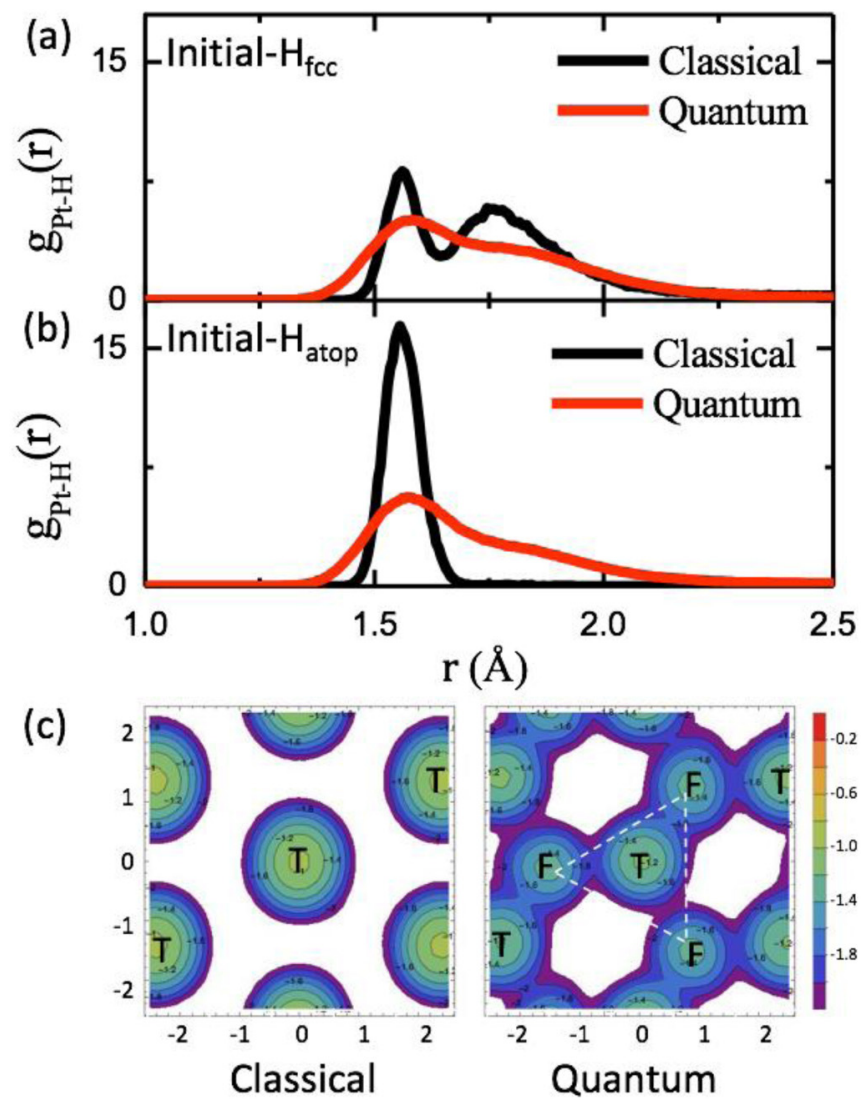

FIG. 2. Pt-H pair distribution for classical (black line) and quantum (red line) simulations with the initial geometry of $\mathrm{H}_{\mathrm{fcc}}$ (a) and $\mathrm{H}_{\text {atop }}$ (b) in vacuum. (c) Hydrogen density profile for the classical and quantum simulations, where $F$ and $T$ represent $\mathrm{H}_{\text {fcc }}$ and $\mathrm{H}_{\text {atop }}$, respectively. The coordinates are in unit of $\AA$. Contours are drawn for the values $10^{-p}$, with $p=-1.8,-1.4, \ldots-0.2$. 
results imply again the coexistence of $\mathrm{H}_{\text {fcc }}$ and $\mathrm{H}_{\text {atop }}$ for the quantum case. The effect of water was thus found to increase the energy barrier existing between the two sites. Note that the coexisting property is in support of some of the experimental papers [3].

Anharmonic effect. We further analyze the nuclear quantum effect using the energy averaged over the trajectories of classical and quantum simuations. For this purpose, we first calculate the adsorption energy at zero temperature as

$$
E_{\mathrm{ads}}^{\mathrm{opt}}=\frac{1}{m}\left[E_{\mathrm{tot}}^{\mathrm{opt}}(\mathrm{Pt})-E_{\mathrm{tot}}^{\mathrm{opt}}(m \mathrm{H} @ \mathrm{Pt})\right]+\frac{1}{2} \mu\left(\mathrm{H}_{2}\right),
$$

where $E_{\mathrm{tot}}^{\mathrm{opt}}(\mathrm{Pt})$ and $E_{\mathrm{tot}}^{\mathrm{opt}}(m \mathrm{H} @ \mathrm{Pt})$ are, respectively, the total energy of Pt surface and that of $m$ hydrogen atom-adsorbed surface calculated with the optimized structures. $\mu\left(\mathrm{H}_{2}\right)$ is the Gibbs free energy per $\mathrm{H}_{2}$ molecule at zero temperature, which is determined from the binding energy of $\mathrm{H}$. The results are shown in Table S1 [48]. Similarly, we also calculate the adsorption energy of classical or quantum simulations at room temperature from the average over $a b$ initio MD or PIMD simulations, respectively, as

$$
\begin{aligned}
E_{\mathrm{ads}}^{\mathrm{md} / \mathrm{pimd}}= & \frac{1}{m}\left[E_{\mathrm{tot}}^{\mathrm{md} / \mathrm{pimd}}(\mathrm{Pt})-E_{\mathrm{tot}}^{\mathrm{md} / \mathrm{pimd}}(m \mathrm{H} @ \mathrm{Pt})\right] \\
& +\frac{1}{2} \mu\left(\mathrm{H}_{2}\right),
\end{aligned}
$$

where $\mu\left(\mathrm{H}_{2}\right)$ is the Gibbs free energy per $\mathrm{H}_{2}$ molecule at room temperature and should be different from the one used in the zero temperature formula [Eq. (3)]. The actual value is, however, irrelevant when comparing the energy at different adsorption sites and it is more convenient to use a common value for our comparative study. We used, therefore, the zero temperature value of the energy throughout.

When the surface is exposed to the vacuum, $\mathrm{H}_{\text {atop }}$ is more stable than $\mathrm{H}_{\mathrm{fcc}}$ by $\sim 40 \pm 9 \mathrm{meV}$ for $E_{\mathrm{ads}}^{\mathrm{opt}}$ and $E_{\mathrm{ads}}^{\mathrm{md}}$, whereas the value of $E_{\text {ads }}^{\text {pimd }}$ is almost the same both for $\mathrm{H}_{\text {fcc }}$ and $\mathrm{H}_{\text {atop }}$ as shown in Table I. These results were used above to conclude that $\mathrm{H}_{\text {atop }}$ dominates in the classical simulation while $\mathrm{H}_{\text {atop }}$ and $\mathrm{H}_{\mathrm{fcc}}$ coexist in the quantum case. The statistical error is estimated from the block averages as less than $15 \mathrm{meV}$. To estimate accurately the adsorption energy, we correct $E_{\mathrm{ads}}^{\text {pimd }}$ by adding the difference between the RPA and PBE energies, which were obtained in our previous study [13]. The corrected values are $\sim 283$ and $264 \mathrm{meV}$, respectively for $\mathrm{H}_{\mathrm{fcc}}$ and $\mathrm{H}_{\text {atop }}$, which are found close to the experimental value $(\$ 270 \mathrm{meV})$ [49]. In addition, we note that the value of $E_{\mathrm{ads}}^{\text {pimd }}$ is similar between the water bilayer and the surface. This explains why the quantum simulations show a similar dynamical behavior in these different environments.

The quantum nuclear effect can be estimated from $\Delta E_{\text {ads }}^{\mathrm{q}}=$ $E_{\mathrm{ads}}^{\mathrm{pimd}}-E_{\mathrm{ads}}^{\mathrm{md}}$ and temperature effect can be estimated from $\Delta E_{\mathrm{ads}}^{\mathrm{T}}=E_{\mathrm{ads}}^{\mathrm{md}}-E_{\mathrm{ads}}^{\mathrm{opt}}$. The negative values in Table I imply that both effects weaken the hydrogen adsorption. The statistical error is estimated from the block averages as less than 20 $\mathrm{meV}$ for the respective quantities (Table I). The temperature effect is almost the same for $\mathrm{H}_{\mathrm{fcc}}$ and $\mathrm{H}_{\text {atop }}$ and is unaffected by the water bilayer. On the contrary, $\Delta E_{\text {ads }}^{\mathrm{q}}$ is smaller for $\mathrm{H}_{\text {atop }}$ than for $\mathrm{H}_{\mathrm{fcc}}$ by about $40 \pm 23 \mathrm{meV}$, indicating that the nuclear quantum effect weakens more the adsorption of
$\mathrm{H}_{\text {atop }}$. According to previous electronic structure calculations, $\mathrm{H}_{\text {atop }}$ is strongly bonded to the surface with a single Pt-H bond compared to $\mathrm{H}_{\mathrm{fcc}}$ that is bound by three bonds, resulting in larger ZPE for $\mathrm{H}_{\text {atop }}$. This is similarly the case in our PIMD simulation. The larger quantum effect is reflected in the pair distribution in Fig. S2 [48], as we could see that the Pt- $\mathrm{H}_{\mathrm{fcc}}$ distribution is broader for the quantum case than for classical case and the difference in the broadening is more conspicuous for Pt- $\mathrm{H}_{\text {atop }}$.

The harmonic and anharmonic terms in the nuclear quantum effect can be estimated in the following manner. The harmonic contribution to the adsorption energy is defined as

$$
\Delta E_{\mathrm{ads}}^{\mathrm{H}}=\frac{1}{m}\left[E^{\mathrm{H}}(\mathrm{Pt})-E^{\mathrm{H}}(m \mathrm{H} @ \mathrm{Pt})\right]+\frac{1}{2} E^{\mathrm{H}}\left(\mathrm{H}_{2}\right),
$$

where $E^{\mathrm{H}}=\sum_{i}\left(\hbar \omega_{i} / 2\right) \operatorname{coth}\left(\hbar \omega_{i} / 2 k_{B} T\right)$ is the nuclear quantum effect in the harmonic approximation, and $\omega_{i}$ is the vibrational frequency calculated by the energy curvature with respect to the displacement of the adsorbed $\mathrm{H}$ and surface $\mathrm{Pt}$ atoms both in the surface normal and parallel directions. $\Delta E_{\text {ads }}^{\mathrm{H}}$ is larger for $\mathrm{H}_{\mathrm{fcc}}$ than for $\mathrm{H}_{\text {atop }}$ by about $50 \mathrm{meV}$ both for the vacuum and solvated surfaces; note that this value is very close to the difference in ZPE's [10,13], indicating that temperature does not affect so much the difference. The adsorption energy in the harmonic approximation is $E_{\mathrm{ads}}^{\mathrm{H}}=$ $\Delta E_{\mathrm{ads}}^{\mathrm{H}}+E_{\mathrm{ads}}^{\mathrm{opt}}$, and the anharmonic contribution can be obtained by subtracting $E_{\text {ads }}^{\mathrm{H}}$ from the quantum adsorption energy as

$$
\Delta E_{\mathrm{ads}}^{\mathrm{AH}}=E_{\mathrm{ads}}^{\mathrm{pimd}}-E_{\mathrm{ads}}^{\mathrm{H}},
$$

which is negative (Table I) indicating that the anharmonic effect also weakens the hydrogen adsorption. The difference between $\mathrm{H}_{\mathrm{fcc}}$ and $\mathrm{H}_{\text {atop }}$ for $\Delta E_{\mathrm{ads}}^{\mathrm{AH}}$ is $15 \pm 13 \mathrm{meV}$ when exposed to the vacuum, while the difference is $1 \pm 17 \mathrm{meV}$ when interfaced with the water bilayer. Compared with the difference for $\Delta E_{\text {ads }}^{\mathrm{H}}$ of $50 \mathrm{meV}$, the harmonic effect is larger and more important for the relative abundance of $\mathrm{H}_{\mathrm{fcc}}$ to $\mathrm{H}_{\text {atop. }}$. Nevertheless, the value for $\Delta E_{\mathrm{ads}}^{\mathrm{AH}}$ is larger than thermal energy $(25 \mathrm{meV})$, and hence the anharmonic effect is not negligible.

Adsorption at coverage of $2 / 3$. We also analyze the result for $\theta=2 / 3$, which has been believed stable near $0 \mathrm{~V}$ vs the standard hydrogen electrode $[2,5]$ and thus very important electrochemically. For the surface exposed to the vacuum, the classical simulation of the initial- $\mathrm{H}_{\mathrm{fcc}}$ showed two peaks at 1.56 and $1.79 \AA$ for the Pt-H pair distributions [Fig. 3(a)], which indicates the coexistence of atop and hollow sites $\left(\mathrm{H}_{\mathrm{fcc}}\right.$ and $\mathrm{H}_{\mathrm{hcp}}$ ) as is clearly shown in Fig. 3(b). Note that the result is different from the one obtained for the full coverage where $\mathrm{H}_{\text {atop }}$ dominates. For the quantum simulation, the Pt-H pair distribution displays only one peak at $1.79 \AA$ and the appearance of the hollow sites in the density profile [Fig. 3(b)]. These density profiles in Fig. 3 have been symmetrized because the symmetry is broken within the finite simulation time. Pt-H pair distribution for the simulation of the initial- $\mathrm{H}_{\text {atop }}$ (Fig. S3 [48]) has a similar behavior to that of the initial- $\mathrm{H}_{\mathrm{fcc}}$ [Fig. 3(a)]. By comparing with the result obtained at the full coverage, we conclude that the concentration of $\mathrm{H}_{\text {atop }}$ is increased as the $\mathrm{H}^{*}$ coverage is increased; this is consistent with the electrochemical experiment where $\mathrm{H}_{\text {atop }}$ can be found 

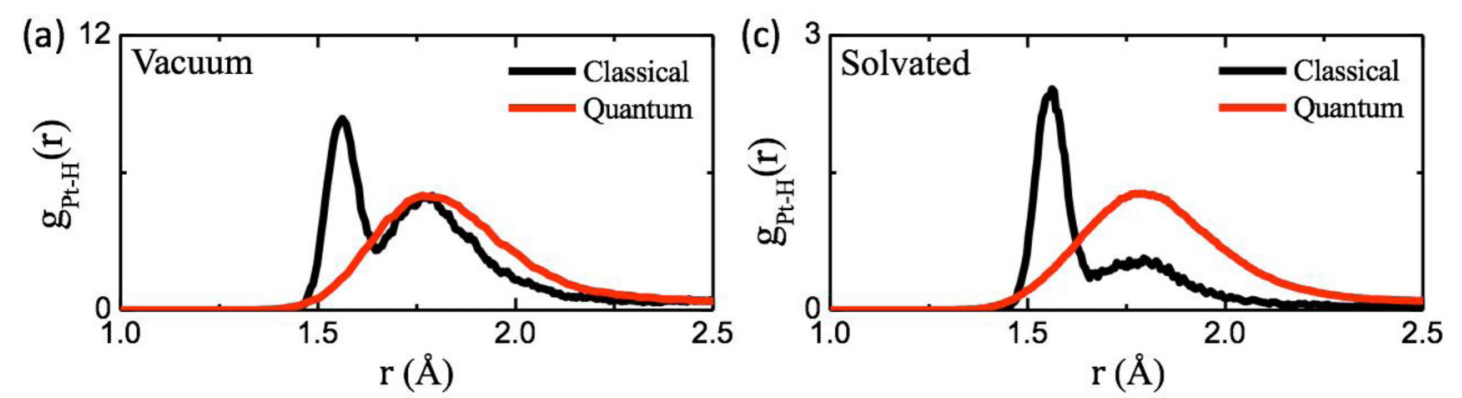

(b)

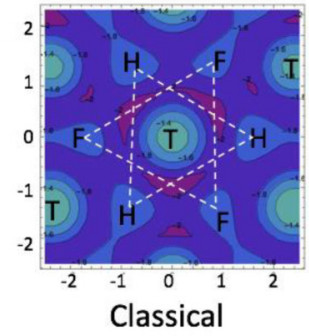

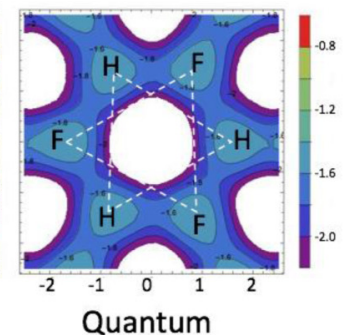

(d)

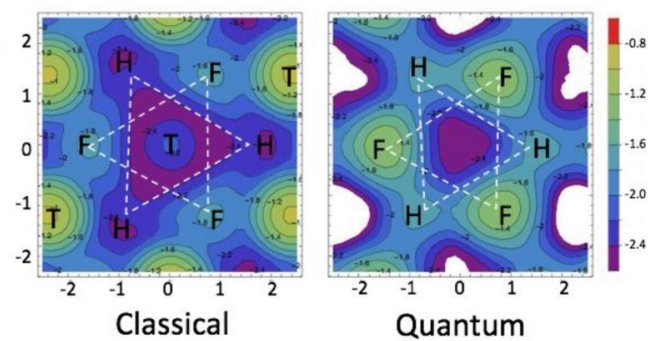

FIG. 3. Pt-H pair distribution for $\mathrm{H} / \mathrm{Pt}(111)$ with initial- $\mathrm{H}_{\mathrm{fcc}}$ on the vacuum (a) and solvated surfaces (c), and corresponding hydrogen density profile for (b) and (d), where $F, T, H$ represent $\mathrm{H}_{\mathrm{fcc}}, \mathrm{H}_{\mathrm{atop}}$, and $\mathrm{H}_{\mathrm{hcp}}$, respectively.

only below the reversible potential [3]. Relatedly, the coverage of atop site increases as the potential on polycrystalline $\mathrm{Pt}$ surface is decreased [50].

When the surface is interfaced with the water bilayer, the $\mathrm{Pt}-\mathrm{H}$ pair distribution shows the peak at the positions obtained when the surface exposed to the vacuum [Fig. 3(c)]. Note that the honeycomb structure of the water bilayers is maintained during the simulation. The presymmetrized density profiles in Fig. S4 [48] show distributions similar to the symmetrized ones. In the classical simulation for the initial- $\mathrm{H}_{\mathrm{fcc}}, \mathrm{H}^{*}$ transferred to the atop sites and remained there until the end of the simulation, which indicates that the atop site is significantly lowered opposed to the case of vacuum. However, the quantum simulation shows only minor distribution on the atop site and the overall profile is similar to that of vacuum. The shape of the Pt-H pair distributions is also similar to that of vacuum [Fig. 3(d)]. We thus conclude that effect of the water bilayer is rather minor for the quantum simulation, which is quite a contrast to the classical case.

It is worth discussing the coverage dependence on the distribution profile. When $\theta=1$, all the hydrogen atoms are located on one of the adsorption sites, atop or fcc, to maximally reduce the strong $\mathrm{H}-\mathrm{H}$ repulsion. When $\theta=2 / 3$, on the contrary, hydrogen atoms are made mobile because of the existing vacant sites. In the quantum simulation, hydrogens move from the fcc site to the hcp site and vice versa through the bridge site, as can be seen from the density profile smeared out at and around the hollow sites. Thereby, dominant sites changed from the atop and fec sites at $\theta=1$ to the fcc and hcp sites at $\theta=2 / 3$. One reason for this drastic change in the mobility could be the quantum effect due to the lowering of kinetic energy by delocalization; this happens when the transfer integral with respect to the fcc-hcp hops becomes significantly large because of their small distance (1.6 ̊). In this case the $\mathrm{H}$ system can be regarded as a correlated quantum liquid when the transfer is at a medium or a low coverage, as is the case for hydrogen on $\operatorname{Pd}(111)$ [51]. Another reason for the mobility is the temperature effect. Since $\mathrm{H}^{*}$ transfers between the hollow sites also in the classical simulation albeit staying most of the time at the atop site, it is possible that the hollow sites can also be occupied by the entropic contribution as the temperature is increased. Indeed, we find that the $\mathrm{H}_{\text {atop }}$ and $\mathrm{H}_{\text {hollow }}$ coexist in the classical simulation at $\theta=2 / 3$, while only $\mathrm{H}_{\text {atop }}$ exists at $\theta=1$. We cannot estimate the relative importance of the two contributions only from the present simulations and thus leave the conclusion to future study on systematic investigation of the temperature dependence. In either case, the lowering in the energy by increasing the mobility is observed for the $\mathrm{H} / \mathrm{Pt}(111)$ system; we consider that this is a typical phenomenon common to strongly correlated hard sphere systems, where particles are localized at a site forming a close packed structure when squeezed, while being more delocalized and made mobile as vacancies are introduced.

Wave function method. Adatoms on a metal, particularly for hydrogen on $\mathrm{Pt}(111)$, are often described using the lattice gas model. To try to interpret our first-principles quantum simulation, let us introduce a transfer integral to the lattice gas model. Within the Born-Oppenheimer approximation, a hydrogen atom is comprised of proton and bound electron and thus is a spinless particle. The $1 \mathrm{~s}$ core electrons of adsorbed $\mathrm{H}$ is tightly bound to a proton. They are not delocalized. Hydrogen localization has been demonstrated from the vibrational spectrum for $\mathrm{H} / \mathrm{Cu}(110)$ [52]. For this purpose, we will use the extended boson-Hubbard model. Gan et al. and Wessel have independently shown that hard sphere bosons on a honeycomb lattice form a solid phase under the full monolayer occupation and transition to a superfluid phase as the chemical potential is reduced at $0 \mathrm{~K}[53,54]$. The radius of the hard sphere was assumed to be equal to the distance between the nearest neighbors (NNs) in those studies, while the radius of $\mathrm{H}$ on $\operatorname{Pt}(111)$ in our study is equal to the distance between the next 
NNs, namely the nearest fcc sites or the nearest hcp sites. Here we use the extended boson-Hubbard model to study further the nature of the transition at finite temperatures.

In the extended boson-Hubbard model, we assign an orbital to each hydrogen atom localized at a site of atop (t), fcc (f) or hcp (h), and the wave function is then described by a linear combination of the product of the orbitals as

$$
|\Psi\rangle_{i}=\sum_{\sigma \in\{\mathrm{t}, \mathrm{f}, \mathrm{h}\}, n \in \operatorname{site}} a_{\sigma_{1} n_{1} \sigma_{2} n_{2} \ldots \sigma_{N} n_{N}, i} \prod_{l=1}^{N} \hat{c}_{\sigma_{l} n_{l}}^{+}|0\rangle .
$$

Note that we have used a Bosonic commutation relation of the operators and neglected the spin degrees of freedom. The Hamiltonian will be approximately

$$
\begin{aligned}
\hat{\mathcal{H}}= & \sum_{\sigma \in\{\mathrm{t}, \mathrm{f}, \mathrm{h}\}, n \in \text { site }} \varepsilon_{\sigma} \hat{n}_{\sigma n}+\sum_{n, n^{\prime} \in\{\text { n.n.of } n\}} t_{\mathrm{fh}}\left(\hat{c}_{\mathrm{f} n}^{+} \hat{c}_{\mathrm{h} n^{\prime}}+\hat{c}_{\mathrm{h} n^{\prime}}^{+} \hat{c}_{\mathrm{f} n}\right) \\
& +\sum_{\sigma, \sigma^{\prime} \neq \sigma, n, n^{\prime} \in\{\text { n.n.of } n\}} U_{\sigma \sigma^{\prime}} \hat{n}_{\sigma n} \hat{n}_{\sigma^{\prime} n^{\prime}} \\
& +\sum_{\sigma, n, n^{\prime} \in\{\text { next n.n.of } n\}} V_{\sigma \sigma} \hat{n}_{\sigma n} \hat{n}_{\sigma n^{\prime}}
\end{aligned}
$$

where the first term is the on-site energy, the second term is the transfer energy between the nearest (n.n.) sites of fcc and hcp, the third term is the repulsion energy of the nearest neighbor pairs, and the last term is the repulsion energy of the next nearest neighbor pairs. The on-site energy includes the zero-point energy, the repulsion energy in the last term were determined referring to the previous study [11], and the typical parameters are set as $\varepsilon_{\mathrm{t}}=-600 \mathrm{meV}, \varepsilon_{\mathrm{f}}=\varepsilon_{\mathrm{h}}=-500 \mathrm{meV}$, $V_{\mathrm{tt}}=50 \mathrm{meV}$, and $V_{\mathrm{ff}}=V_{\mathrm{hh}}=30 \mathrm{meV}$. The third term can be taken to be infinity according to the ab initio calculations. Therefore, the transfer energy $t_{\mathrm{fh}}$ is the unknown parameter in this study. Note that, without the transfer, it is equivalent to a lattice gas model and has been studied previously [11].

We use a $3 \times 3$ cell and apply the periodic boundary condition with a $\Gamma$ point only. The model consists of nine sites for each atop, fcc, and hcp site. The sites are occupied by $3 \sim 8$ hydrogen atoms and the hydrogen coverage becomes $\theta=$ $3 / 9 \sim 8 / 9$. The exact diagonalization of the Hamiltonian is an easy task, e.g., there are only about 1000 configurations at the coverage $6 / 9$ because most configurations are prohibited by the infinite repulsion between the nearest neighbor pairs. The occupation profile at finite temperature can be estimated from the wave function as

$$
\begin{aligned}
P_{\sigma n}= & \sum_{\sigma_{1} n_{1} \ldots \sigma_{N} n_{N}, i}\left|a_{\sigma_{1} n_{1} \sigma_{2} n_{2} \ldots \sigma_{N} n_{N}, i}\right|^{2}\left(\delta_{\sigma, \sigma_{1}} \delta_{n, n_{1}}+\cdots\right. \\
& \left.+\delta_{\sigma, \sigma_{N}} \delta_{n, n_{N}}\right) \exp \left[-\frac{E_{i}}{k_{B} T}\right]
\end{aligned}
$$

and then we obtained the occupation profile for atop, fcc, and hcp at room temperature labeled as $P_{\mathrm{t}}, P_{\mathrm{f}}, P_{\mathrm{h}}$ by summing over all the sites $n$. Relative abundance of the atop site can be defined as $R_{\mathrm{t}}=\left(P_{\mathrm{t}}-P_{\mathrm{f}}-P_{\mathrm{h}}\right) /\left(P_{\mathrm{t}}+P_{\mathrm{f}}+P_{\mathrm{h}}\right) \cdot R_{\mathrm{t}}=1$ and -1 indicates that all the hydrogen atoms adsorbed on the atop and hollow sites, respectively. Near the full coverage $8 / 9$ and $7 / 9$, hydrogen distribution is independent of the transfer energy $t_{\text {fh }}$ as shown in Fig. 4, which was obtained

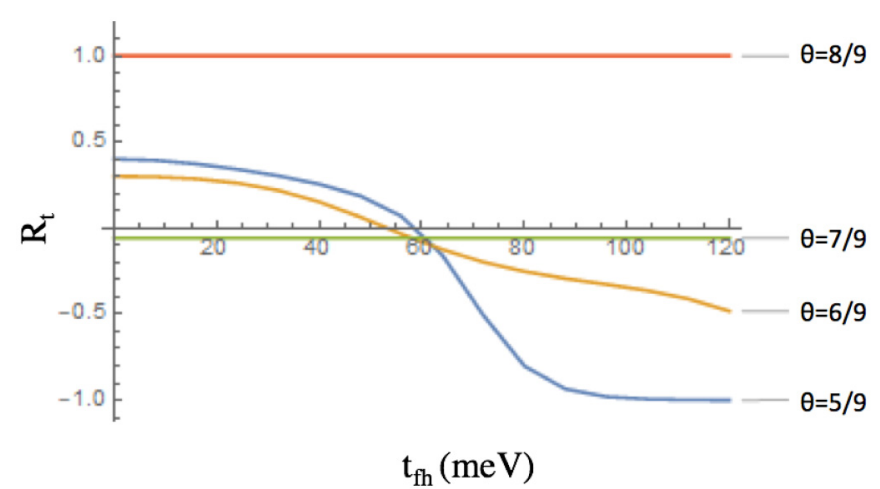

FIG. 4. Relative abundance of the atop site $R_{\mathrm{t}}$ against the values of the transfer integral $t_{\mathrm{fh}}$ from the quantum lattice gas model.

using the typical parameters for the on-site and repulsion as shown above. When the coverage is decreased to $5 / 9$ and $6 / 9, \mathrm{H}_{\text {atop }}$ dominates without the fcc-hcp transfer $\left(t_{\mathrm{fh}}=0\right)$. As the transfer energy $t_{\mathrm{fh}}$ is increased, the distributions for $\mathrm{H}_{\text {atop }}$ is decreased while that for the hollow sites are increased. When $t_{\mathrm{fh}}$ reaches $120 \mathrm{meV}$ for $\theta=5 / 9$, the population is totally on the hollow sites. The cases of the $\theta=3 / 9$ and $4 / 9$ have a similar dependence on the $t_{\mathrm{fh}}$ as shown in Fig. S5 [48]. Note that the results for low coverages can be compared with a forgoing research done at $\theta=1 / 4$, where the threedimensional Schrödinger equation was solved for hydrogen on a DFT potential energy surface. The wave function was found localized around the hollow sites [14,55]. From Fig. 4, we conjecture that there is a critical coverage around 0.7 below which the dominant site depends on the value of the transfer. Below the critical value, an fcc-hcp transfer plays a role in stabilizing the hollow sites, which naturally explains why only the hollow sites appearing at the coverage $2 / 3$ in the $a b$ initio PIMD simulations.

Vibrational spectrum. Vibrational spectrum of the hydrogenated $\mathrm{Pt}(111)$ surface is a means for identifying the adsorption site. Using the trajectories of ab initio RPMD and ab initio MD simulations, we have computed the spectra by the Fourier transform of the dipole autocorrelation function. In ab initio RPMD we used the formula [56]

$$
\alpha(\omega)=\frac{\omega^{2}}{2 \pi} \int_{-\infty}^{\infty} d t e^{-i \omega t}\left\langle\mathbf{M}_{c}(0) \mathbf{M}_{c}(t)\right\rangle,
$$

where $M_{c}(t)=\frac{1}{P} \sum_{s=1}^{P} M^{(s)}(t)$ is the bead-averaged dipole moment in the surface normal direction, which are directly obtained from electronic structure calculation at time $t$. The results are displayed in Fig. 5. When performing the analysis of the quantum (classical) simulation done under the vacuum using the time domain where the hydrogen atoms are adsorbed on fcc, the resulting curve has a peak at about $160 \mathrm{meV}$ $(168 \mathrm{meV})$ at coverage 1 . The quantum simulation yields a peak $170 \mathrm{meV}$ at coverage $2 / 3$. The peak at $160 \mathrm{meV}$ can be compared with the experimental one at $\sim 153 \mathrm{meV}$, which was identified as the symmetric stretch mode at the fcc site with the help of the calculated adiabatic potential energy surface [57]. In the experiment, additional peaks were observed at $31 \mathrm{meV}$ assigned as dipole-active vibration and $113 \mathrm{meV}$ as a hybrid mode with both in-plane and dipole characters. 


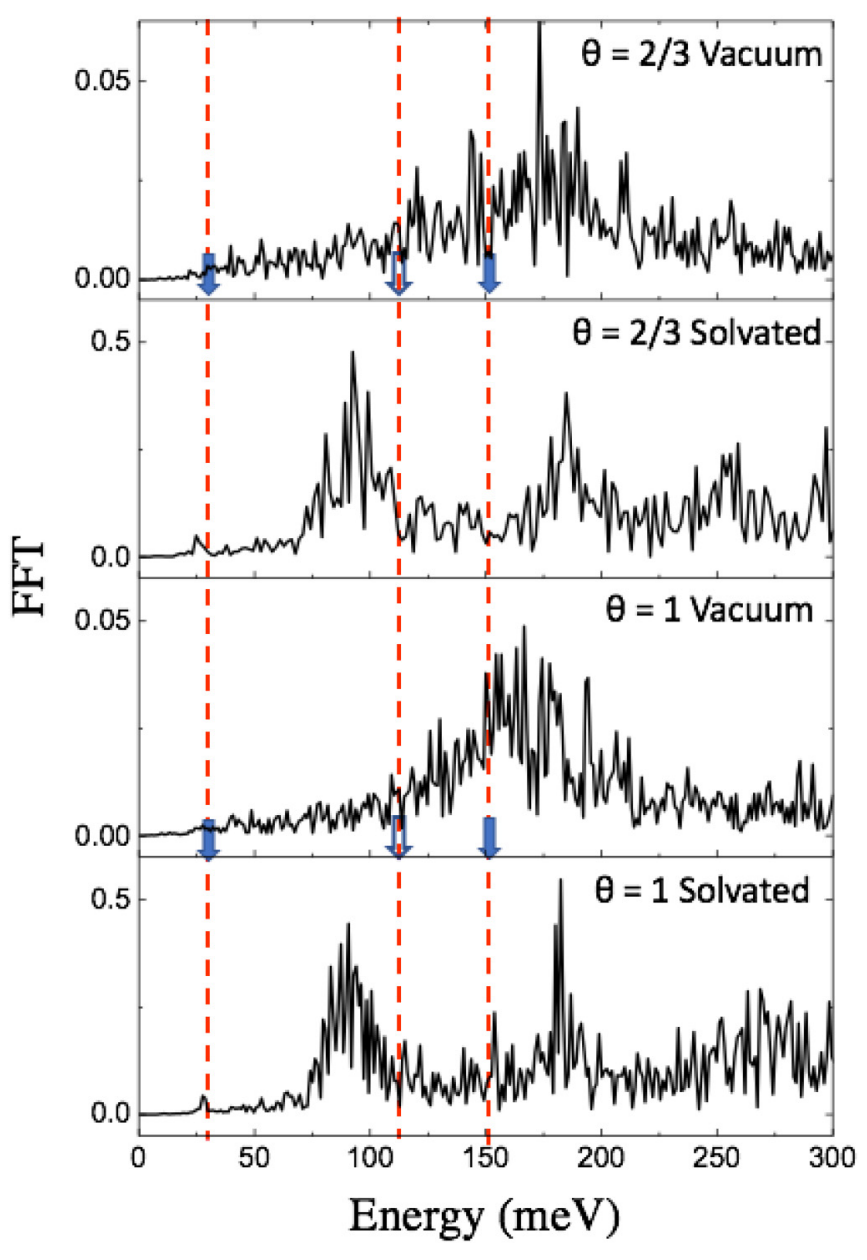

FIG. 5. Fourier transform (FFT) of the dipole moment in the surface normal direction for $\mathrm{H}_{\mathrm{fcc}}$ at $\theta=1$ and $2 / 3$ in vacuum and solvated case. The vertical dashed line pointed out the experimental value for vacuum case from Ref. [57].

While corresponding peaks are not apparent in the vacuum case, peaks are found at 25 and $90 \mathrm{meV}$ in the quantum simulation done with the water bilayer, which may possibly correspond to the experimental peaks at 31 and $113 \mathrm{meV}$, respectively. When the classical trajectory analysis is applied to $\mathrm{H}_{\text {atop }}$, we obtained the peak at $277 \mathrm{meV}$, which can be compared with the experimental value $260 \mathrm{meV}$ obtained for a polycrystalline Pt [50]. The experimental peak heights are washed away when the coverage is reduced below about 0.7 [57], while the coverage dependence is weak in our simulation presumably because much lower temperature $(85 \mathrm{~K})$ is set at the experiment. We note that the peaks in the solvated case is sharper than that in vacuum in Fig. 5. It is likely that the water tends to localize the adsorbed hydrogen atoms and may play a significant role in determining the vibrational frequency.

\section{SUMMARY}

We have applied $a b$ initio MD, PIMD, and RPMD simulations to the $\mathrm{H} / \mathrm{Pt}(111)$ system to reveal the interplays of the nuclear quantum effect and the strong $\mathrm{H}-\mathrm{H}$ repulsion, which induces and hampers, respectively, delocalization over the nearly degenerate adsorption sites. At the full monolayer condition, $\mathrm{H}$ atoms are close-packed either at the atop sites or the fcc sites because of the repulsion, while at the $2 / 3$ monolayer condition, $\mathrm{H}$ atoms have room for the hopping and are delocalized over the fcc and hcp sites via the bridge site, as do particles in quantum liquids. The crossover of the site occupation, thus reflecting the quantum many-body nature of the system, is a clue for reconciling the controversies of the experiments. The effect of the solution on the electrochemical interface was further investigated by attaching the water bilayers. The solution was found to play a minor role on the most stable adsorption sites but significantly affected the diffusion and the vibration. This effect may also be a key to understanding the electrochemical processes like hydrogen evolution reaction.

\section{ACKNOWLEDGMENTS}

The calculations were performed on the supercomputers at Institute for Solid State Physics, the University of Tokyo and Research Center for Computational Science, Okazaki Research Facilities, National Institutes of Natural Sciences, and National Institute for Materials Science. This research was supported by MEXT as "Priority Issue on Post-K computer (Supercomputer Fugaku)" (Development of new fundamental technologies for high-efficiency energy creation, conversion/storage and use) using computational resources of the K computer provided by the RIKEN Center for Computational Science, and other HPCI System through the HPCI System Research project (Project ID: hp170241, hp160225, hp180209, and hp190174) and "Development of Environmental Technology using Nanotechnology" program. We are also supported by Grants in Aid for Scientific Research on Innovative Area "Hydrogenomics" (Grant No. 18H05519) from the Japan Society for the Promotion of Science (JSPS). M.S. acknowledges JSPS (Grants No. 18H01693, No.18K05208, and No. 16K05675) for financial support and JAEA for the usage CCSE supercomputers.
[1] B. Hammer and J. K. Nørskov, Electronic factors determining the reactivity of metal surfaces, Surf. Sci. 343, 211 (1995).

[2] N. M. Marković and P. N. Ross Jr., Surface science studies of model fuel cell electrocatalysts, Surf. Sci. Rep. 45, 117 (2002).

[3] G. Jerkiewicz, Electrochemical hydrogen adsorption and absorption. Part 1: Under-potential deposition of hydrogen, Electrocatal. 1, 179 (2010).
[4] A. R. Zeradjanin, J. P. Grote, G. Polymeros, and K. J. J. Mayrhofer, A critical review on hydrogen evolution electrocatalysis: Re-exploring the volcano-relationship, Electroanalysis 28, 2256 (2016).

[5] G. Jerkiewicz, Hydrogen sorption at/in electrodes, Prog. Surf. Sci. 57, 137 (1998).

[6] A. Peremans and A. Tadjeddine, Electrochemical deposition of hydrogen on platinum single crystals studied by 
infrared-visible sum-frequency generation, J. Chem. Phys. 103, 7197 (1995).

[7] Ş. Bădescu, P. Salo, T. Ala-Nissila, S. C. Ying, K. Jacobi, Y. Wang, K. Bedürftig, and G. Ertl, Energetics and Vibrational States for Hydrogen on Pt(111), Phys. Rev. Lett. 88, 136101 (2002).

[8] I. Hamada and Y. Morikawa, Density-functional analysis of hydrogen on $\mathrm{Pt}(111)$ : Electric field, solvent, and coverage effects, J. Phys. Chem. C 112, 10889 (2008).

[9] P. J. Feibelman, First-principles calculations of stress induced by gas adsorption on Pt (111), Phys. Rev. B 56, 2175 (1997).

[10] T. L. Tan, L.-L. Wang, D. D. Johnson, and K. Bai, Hydrogen deposition on $\operatorname{Pt}(111)$ during electrochemical hydrogen evolution from a first-principles multiadsorption-site study, J. Phys. Chem. C 117, 22696 (2013)..

[11] T. T. T. Hanh, Y. Takimoto, and O. Sugino, First-principles thermodynamic description of hydrogen electroadsorption on the Pt(111) surface, Surf. Sci. 625, 104 (2014).

[12] L. Schimka, J. Harl, A. Stroppa, A. Grüneis, M. Marsman, F. Mittendorfer, and G. Kresse, Accurate surface and adsorption energies from many-body perturbation theory, Nat. Mater. 9, 741 (2010).

[13] L. Yan, Y. Sun, Y. Yamamoto, S. Kasamatsu, I. Hamada, and O. Sugino, Hydrogen ddsorption on $\mathrm{Pt}(111)$ revisited from random phase approximation, J. Chem. Phys. 149, 164702 (2018).

[14] G. Källén and G. Wahnström, Quantum treatment of H adsorbed on a Pt(111) surface, Phys. Rev. B 65, 033406 (2001).

[15] R. P. Feynman, Statistical Mechanics: A Set of Lectures (Hachette, UK, 1998).

[16] R. P. Feynman, A. R. Hibbs, and D. F. Styer, Quantum Mechanics and Path Integrals (Courier Corporation, New York, 2010).

[17] D. Chandler and P. G. Wolynes, Exploiting the isomorphism between quantum theory and classical statistical mechanics of polyatomic fluids, J. Chem. Phys. 74, 4078 (1981).

[18] M. Tuckerman, Statistical Mechanics: Theory and Molecular Simulation (Oxford University Press, Oxford, 2010).

[19] M. Shiga, Path integral simulations, Reference Module in Chemistry, Molecular Sciences and Chemical Engineering (Elsevier, 2018).

[20] I. R. Craig and D. E. Manolopoulos, Quantum statistics and classical mechanics: Real time correlation functions from ring polymer molecular dynamics, J. Chem. Phys. 121, 3368 (2004).

[21] D. Marx and M. Parrinello, Ab initio path integral molecular dynamics: Basic ideas, J. Chem. Phys. 104, 4077 (1996).

[22] D. Marx and J. Hutter, Ab Initio Molecular Dynamics: Basic Theory and Advanced Methods (Cambridge University Press, Cambridge, 2009).

[23] M. Shiga, M. Tachikawa, and S. Miura, Ab initio molecular orbital calculation considering the quantum mechanical effect of nuclei by path integral molecular dynamics, Chem. Phys. Lett. 332, 396 (2000).

[24] M. Shiga, M. Tachikawa, and S. Miura, A unified scheme for ab initio molecular orbital theory and path integral molecular dynamics, J. Chem. Phys. 115, 9149 (2001).

[25] M. Shiga and A. Nakayama, Ab initio path integral ring polymer molecular dynamics: Vibrational spectra of molecules, Chem. Phys. Lett. 451, 175 (2008).

[26] Y. Kawashima and M. Tachikawa, Ab Initio path integral molecular dynamics study of the nuclear quantum effect on out-of- plane ring deformation of hydrogen maleate anion, J. Chem. Theory Comput. 10, 153 (2014).

[27] M. Machida, K. Kato, and M. Shiga, Nuclear quantum effects of light and heavy water studied by all-electron first principles path integral simulations, J. Chem. Phys. 148, 102324 (2018).

[28] H. Kimizuka, S. Ogata, and M. Shiga, Unraveling anomalous isotope effect on hydrogen diffusivities in fcc metals from first principles including nuclear quantum effects, Phys. Rev. B 100 , 024104 (2019).

[29] H. Kimizuka, S. Ogata, and M. Shiga, Mechanism of fast lattice diffusion of hydrogen in palladium: Interplay of quantum fluctuations and lattice strain, Phys. Rev. B 97, 014102 (2018).

[30] D. Kang, H. Sun, J. Dai, W. Chen, Z. Zhao, Y. Hou, J. Zeng, and J. Yuan, Nuclear quantum dynamics in dense hydrogen, Sci. Rep. 4, 5484 (2014).

[31] X.-Z. Li, B. Walker, and A. Michaelides, Quantum nature of the hydrogen bond, Proc. Natl. Acad. Sci. USA 108, 6369 (2018).

[32] Y. Litman, D. Donadio, M. Ceriotti, and M. Rossi, Decisive role of nuclear quantum effects on surface mediated water dissociation at finite temperature, J. Chem. Phys. 148, 102320 (2018).

[33] S. Ruiz-Barragan, K. Ishimura, and M. Shiga, On the hierarchical parallelization of $a b$ initio simulations, Chem. Phys. Lett. 646, 130 (2016); M. Shiga, PIMD version 2.2, 2018, https://ccse.jaea.go.jp/software/PIMD/index.jp.html.

[34] G. Kresse and J. Furthmuller, Efficient iterative schemes for $a b$ initio total-energy calculations using a plane-wave basis set, Phys. Rev. B 54, 11169 (1996).

[35] P. E. Blöchl, Projector augmented-wave method, Phys. Rev. B 50, 17953 (1994).

[36] J. P. Perdew, K. Burke, and M. Ernzerhof, Generalized Gradient Approximation Made Simple, Phys. Rev. Lett. 77, 3865 (1996).

[37] T. Roman and A. Groß, Structure of water layers on hydrogencovered Pt electrodes, Catal. Today 202, 183 (2013).

[38] M. Kiskinova, G. Pirug, and H. Bonzel, Adsorption and decomposition of $\mathrm{H}_{2} \mathrm{O}$ on a K-covered $\mathrm{Pt}(111)$ surface, Surf. Sci. 150, 319 (1985).

[39] G. J. Martyna, M. L. Klein, and M. Tuckerman, Nosé-Hoover chains: The canonical ensemble via continuous dynamics, J. Chem. Phys. 97, 2635 (1992).

[40] W. G. Hoover, Canonical dynamics: Equilibrium phase-space distributions, Phys. Rev. A 31, 1695 (1985).

[41] S. Nosé, A unified formulation of the constant temperature molecular dynamics methods, J. Chem. Phys. 81, 511 (1984).

[42] W. Shinoda and M. Shiga, Quantum simulation of the heat capacity of water, Phys. Rev. E 71, 041204 (2005).

[43] H. A. Stern, F. Rittner, B. J. Berne, and R. A. Friesner, Combined fluctuating charge and polarizable dipole models: Application to a five-site water potential function, J. Chem. Phys. 115, 2237 (2001).

[44] Generally, the converged number of beads grows with the vibrational frequency. 16 beads were sufficient because the Pt-H stretching mode has highest frequency about $2200 \mathrm{~cm}^{-1}$ (which is much lower than that of the $\mathrm{OH}$ stretching mode of water). 
[45] M. E. Tuckerman, B. J. Berne, and G. J. Martyna, Reversible multiple time scale molecular dynamics, J. Chem. Phys. 97, 1990 (1992).

[46] W. Fang, J. O. Richardson, J. Chen, X. Z. Li, and A. Michaelides, Simultaneous Deep Tunneling and Classical Hopping for Hydrogen Diffusion on Metals, Phys. Rev. Lett. 119, 126001 (2017).

[47] P. S. Rice, Y. Mao, C. Guo, and P. Hu, Interconversion of hydrated protons at the interface between liquid water and platinum, Phys. Chem. Chem. Phys. 21, 5932 (2019).

[48] See Supplemental Material at http://link.aps.org/supplemental/ 10.1103/PhysRevB.101.165414 for Pt-H pair distribution and relative abundance of the atop site obtained from the quantum lattice gas model.

[49] R. Gomez, J. M. Orts, B. Alverez-Ruiz, and J. M. Feliu, Effect of temperature on hydrogen adsorption on $\mathrm{Pt}(111), \operatorname{Pt}(110)$, and $\mathrm{Pt}(100)$ electrodes in $0.1 \mathrm{M} \mathrm{HClO}_{4}$, J. Phys. Chem. B 108, 228 (2004).

[50] K. Kunimatsu, T. Senzaki, G. Samjeské, M. Tsushima, and M. Osawa, Hydrogen adsorption and hydrogen evolution reaction on a polycrystalline Pt electrode studied by surface-enhanced infrared absorption spectroscopy, Electrochimica Acta 52, 5715 (2007).
[51] M. Nishijima, H. Okuyama, N. Takagi, T. Aruga, and W. Brenig, Quantum delocalization of hydrogen on metal surfaces, Surf. Sci. Rep. 57, 113 (2005).

[52] C. Astaldi, A. Bianco, S. Modesti, and E. Tosatti, Vibrational Spectra of Atomic $\mathrm{H}$ and $\mathrm{D}$ on $\mathrm{Cu}(110)$ : Evidence for $\mathrm{H}$ Quantum Delocalization, Phys. Rev. Lett. 68, 90 (1992).

[53] J. Y. Gan, Y. C. Wen, J. Ye, T. Li, S. J. Yang, and Y. Yu, Extended Bose-Hubbard model on a honeycomb lattice, Phys. Rev. B 75, 214509 (2007).

[54] S. Wessel, Phase diagram of interacting bosons on the honeycomb lattice, Phys. Rev. B 75, 174301 (2007).

[55] K. Nobuhara, H. Nakanishi, H. Kasai, and A. Okiji, Behavior of $\mathrm{H}$ atom in adsorption states on metal surfaces -localization and delocalization, Surf. Sci. 493, 271 (2001).

[56] A. Witt, S. D. Ivanov, M. Shiga, H. Forbert, and D. Marx, On the applicability of centroid and ring polymer path integral molecular dynamics for vibrational spectroscopy, J. Chem. Phys. 130, 194510 (2009).

[57] Ş. C. Bădescu, K. Jacobi, Y. Wang, K. Bedürftig, G. Ertl, P. Salo, T. Ala-Nissila, and S. C. Ying, Vibrational states of a $\mathrm{H}$ monolayer on the $\mathrm{Pt}(111)$ surface, Phys. Rev. B 68, 205401 (2003). 